\title{
La enseñanza de la conciencia fonológica en la Educación Infantil: un estudio observacional
}

\author{
Ruth Sánchez-Rivero* y Raquel Fidalgo \\ Dpto. Psicología, Sociología y Filosofía. Universidad de León.
}

\begin{abstract}
Resumen: La conciencia fonológica es clave en el aprendizaje lector inicial y en la explicación de sus dificultades. Parece por tanto necesario proporcionar al alumnado una instrucción empíricamente validada en conciencia fonológica desde la Educación Infantil. El presente estudio analiza la práctica instruccional de 113 docentes de Educación Infantil para el desarrollo de la conciencia fonológica y su ajuste a las evidencias empíricas sobre su efectividad en función de la edad del alumnado. Se realizó una observación participante de la práctica instruccional del profesorado en conciencia fonológica y se elaboró un informe descriptivo atendiendo a los distintos niveles de conciencia fonológica, los diferentes tipos de tareas instruccionales, y los materiales y estrategias que empleaban. Los resultados muestran que, aunque el profesorado analizado instruye en conciencia fonológica, sus planteamientos presentan desajustes en comparación con los conocimientos derivados de la investigación científica. No se ha encontrado relación significativa en función de la edad del alumnado excepto en la instrucción en el nivel de sílaba y el tipo de agrupamientos cuando se trabajan los fonemas. Se discuten los resultados considerando las evidencias científicas en relación a la instrucción en conciencia fonológica y se sugieren líneas de mejora para su enseñanza en la Educación Infantil.

Palabras clave: Conciencia fonológica; Lectura; Prácticas efectivas; Prevención.
\end{abstract}

\section{The teaching of phonological awareness in pre-school education: an observational study}

\begin{abstract}
Phonological awareness plays a key role in students' early reading and the explanation of their learning difficulties. Therefore, it seems necessary to provide students with evidence-based instruction focused on phonological awareness development from the pre-school stage. The present study aims to analyze the instructional practice implemented by 113 pre-school teachers for phonological awareness development and to determine whether it fits the existing empirical evidence about the effectiveness of instruction according to students' age. We observed pre-school teachers' instructional practice of phonological awareness and elaborated a descriptive report including the different levels of phonological awareness, the different types of instructional tasks, and the materials and strategies that the teachers used. Results show that although the teachers were instructing phonological awareness, their instructional practices are not adapted to scientific knowledge. No significant relation was found in the phonological awareness instruction implemented by the teachers in terms of the students' age, except for the instruction at the syllable level and the type of groupings when addressing phonemes. Results are discussed considering the scientific evidence of phonological awareness instruction, and suggestions to improve the teaching of phonological awareness in pre-school are provided.

Keywords: Phonological awareness; Reading; Evidence-based practices; Prevention.
\end{abstract}

La conciencia fonológica se refiere a la capacidad de reflexionar y manipular los diferentes segmentos que componen el

Recibido: 30/07/2019 - Aceptado: 22/04/2020 - Avance online: 01/06/2020 *Correspondencia: Ruth Sánchez-Rivero

Dpto. Psicología, Sociología y Filosofía. Universidad de León. León, España.

C.P: 24071, León, España.

E-mail: rsanr@unileon.es

Sánchez-Rivero, R. y Fidalgo, R. (2020). La enseñanza de la conciencia fonológica en la Educación Infantil: un estudio observacional. Revista de Psicología y Educación, 15(2), 184 200, hitps://doi.org/10.23923/rpye2020.02.195 lenguaje hablado (Defior y Serrano, 2011; Rabazo y Moreno, 2004). Dentro del término conciencia fonológica es posible considerar diferentes niveles en función del segmento específico que se manipule, tales como la rima, la sílaba o los fonemas (Rabazo y Moreno, 2004), incluyendo algunos estudios también el nivel léxico (Defior y Serrano, 
2011). La importancia de la conciencia fonológica en el aprendizaje de la lectura, y en la explicación de sus dificultades, ha sido reflejada en numerosas revisiones científicas como las realizadas por Defior y Serrano (2011), Jiménez y O Shanahan (2008) y Rabazo y Moreno (2004).

Por una parte, el valor predictivo de la conciencia fonológica en el aprendizaje lector inicial se ha corroborado empíricamente tanto en el caso del inglés (Castles y Colthearth, 2004; Fuchs et al., 2012) como en el del castellano (Bravo, Villalón y Orellana, 2006; Suarez-Coalla, García-de-Castro y Cuetos, 2013), si bien, varía en función del grado de transparencia de la lengua (Ziegler, et al., 2010). Específicamente en castellano, Suarez-Coalla et al. (2013) realizaron un estudio centrado en conocer qué variables, evaluadas cuando el alumnado iniciaba el $2^{\circ}$ curso del $2^{\circ}$ ciclo de Educación Infantil (4-5 años), predecían la lectura mecánica evaluada 18 meses después cuando el alumnado se encontraba finalizando el tercer curso del $2^{\circ}$ ciclo de Educación Infantil (5-6 años). Los resultados mostraron que la conciencia fonológica fue la variable que mejor predijo la exactitud lectora cuando el alumnado se encontraba finalizando $3^{\circ}$ de Educación Infantil. Por su parte, Bravo et al. (2006) realizaron un estudio de seguimiento entre $1^{\circ}$ y $3^{\circ}$ de Educación Primaria, centrado en conocer qué variables influían sobre el rendimiento lector de 262 estudiantes que comenzaban la Educación Primaria. Sus resultados mostraron que el reconocimiento del primer fonema fue la variable que mejor predijo el aprendizaje de la lectura en los tres cursos, permitiendo discriminar entre estudiantes con alto y bajo rendimiento lector. Por lo tanto, en castellano, la influencia que ejerce la conciencia fonológica sobre el aprendizaje lector inicial comienza desde la Educación Infantil y se mantiene hasta los primeros cursos de la Educación Primaria.

Por otro lado, estudios de revisión centrados en el aprendizaje de la lectura y sus dificultades, han mostrado que las principales dificultades que presenta el alumnado con dislexia se dan en el dominio de las habilidades de reconocimiento de la palabra, donde se encuentra implicado el procesamiento fonológico y concretamente la conciencia fonológica (Defior y Serrano, 2011 ; Jiménez y O Shanahan, 2008). En este sentido, estudios realizados por Jiménez y colaboradores (Jiménez et al., 2009; Jiménez, Rodríguez, Guzmán y García, 2010) centrados en conocer las diferencias en el desarrollo de los procesos de lectura entre alumnos normolectores y alumnos con dificultades lectoras, mostraron la existencia de diferencias en conciencia fonológica entre ambos grupos, sobre todo hasta $3^{\circ}$ de Educación Primaria, donde pasaron a cobrar mayor importancia otras variables como la velocidad de procesamiento o el acceso al léxico (Jiménez et al., 2009).

Por otra parte, desde un punto de vista instruccional también existen evidencias empíricas que han corroborado la eficacia de instruir en conciencia fonológica sobre el aprendizaje de la lectura, tanto en alumnado con o sin dificultades de aprendizaje. En muestras normalizadas, diversos estudios han mostrado la eficacia de instruir de forma temprana y contextualizada en conciencia fonológica en el $2^{\circ}$ ciclo de Educación Infantil. Específicamente, los estudios que han trabajado con alumnado de 3 y 4 años han mostrado que resulta efectivo proporcionar al alumnado una instrucción en los niveles de rima y sílaba, al menos para favorecer el desarrollo de las habilidades metafonológicas ya que no se obtuvieron efectos sobre la lectura (ver estudios de Arnáiz, Castejón, Ruiz y Guirao, 2002; Gimeno, Clemente, López y Castro, 1994). Por su parte, los estudios desarrollados con alumnado de 5 años se han centrado fundamentalmente en el nivel fonémico, obteniendo mejoras en las habilidades fonológicas y/o en la lectura de palabras y pseudopalabras (ver estudios de Cuadro y Trías, 2008; Domínguez, 1994; 1996a; 1996b; Ramos, 2004; Ramos y Cuadrado, 2003). Asimismo, también se ha puesto de relevancia que, en función de la dimensión de la conciencia fonológica que se trabaje, resulta efectivo instruir a partir de determinadas actividades y materiales. 
Así, para instruir en el nivel de rima y sílaba resultó efectivo el empleo de tareas de identificación y recuento (ya que exigen menores esfuerzos cognitivos por parte del alumnado) y sin necesidad de apoyos visuales o escritos (Gimeno et al., 1994). Respecto a la instrucción en conciencia fonémica, la mayoría de estudios combinan la instrucción en el nivel fonémico con la enseñanza de las reglas de conversión mediante el uso de material manipulable (Defior, 2008; Defior y Tudela, 1994; Domínguez, 1994, 1996a; González, López, Vilar y Rodríguez, 2013; Ramos y Cuadrado, 2003) y mediante tareas de segmentación, identificación, adición y omisión de fonemas (Cuadro y Trías, 2008; Domínguez, 1994, 1996a; González et al., 2013; Ramos y Cuadrado, 2003).

Por otra parte, en el ámbito de las dificultades de aprendizaje de la lectura, se ha mostrado la eficacia de intervenir en conciencia fonémica, junto con el apoyo visual de letras, con alumnado de Educación Primaria (Hernández-Valle y Jiménez, 2001 ; Jiménez y Rojas, 2008). Así, en el estudio desarrollado por Hernández-Valle y Jiménez (2001) con alumnado de $2^{\circ}$ y $3^{\circ}$ de Educación Primaria con dificultades de aprendizaje de la lectura y mediante una intervención en pequeño grupo con dos sesiones semanales de 45 minutos durante tres meses, se obtuvieron mejoras en la lectura de palabras y pseudopalabras. Además, en este estudio también se compararon los efectos de dicha intervención en alumnado de $2^{\circ}$ curso frente al alumnado de tercer curso, obteniéndose que fue el alumnado de $2^{\circ}$ curso el que obtuvo mejores resultados en la lectura de pseudopalabras. Por su parte, en el estudio de Jiménez y Rojas (2008) también se obtuvieron mejoras en la lectura de palabras y pseudopalabras con alumnado de $4^{\circ}$ a $6^{\circ}$ de Educación Primaria con dificultades de aprendizaje de la lectura a través de una intervención individualizada y desarrollada por un especialista con cinco sesiones semanales de 30 minutos cada una durante un mes.

Teniendo en cuenta la influencia que la conciencia fonológica juega en el aprendizaje lector inicial del alumnado y su logro, parece evidente la importancia de que el profesorado en edades tempranas como en la etapa de Educación Infantil proporcione al alumnado una instrucción efectiva que favorezca el desarrollo de la conciencia fonológica, que promueva así mismo una adquisición óptima de la lectura en el alumnado. Dicha necesidad también se ve avalada a nivel legislativo en nuestro país. Así, en la normativa educativa se aboga por un enfoque preventivo y de atención temprana de las dificultades de aprendizaje, cuya responsabilidad recaería en el profesorado de las etapas educativas iniciales (Fidalgo y Robledo, 2010). Este enfoque preventivo es coherente con el planteamiento del modelo de respuesta a la intervención, vigente internacionalmente a nivel científico y normativo, para la identificación de las dificultades de aprendizaje (Fuchs, Fuchs y Compton, 2012; Jiménez, 2010), en línea con la última actualización del DSM5. Este modelo apoya la idea de que las dificultades de aprendizaje no pueden ser diagnosticadas sin una evidencia que determine que el alumnado ha recibido una práctica instruccional efectiva, fundamentada en la evidencia científica. Así, el énfasis se traslada al ámbito instruccional y a la necesidad de que las practicas instruccionales para la enseñanza, en el caso concreto que nos ocupa, de la lectura, cuenten con un aval científico sobre su efectividad (National Joint Committee of Learning Disabilities, 2006). La eficacia de este modelo para la identificación de las dificultades de aprendizaje, ha sido empíricamente demostrada tanto en el aprendizaje de la lectura (Simmons et al., 2008), como de la escritura (Arrimada, Torrance y Fidalgo, 2018) y las matemáticas (Vanderheyden, Witt y Gilbertson, 2007).

En este contexto se enmarca el interés del presente estudio centrado en analizar la práctica instruccional del profesorado para favorecer el desarrollo de la conciencia fonológica del alumnado de Educación Infantil en función del curso (de 3, 4 y 5 años), con el fin de poder detectar en qué medida dichas prácticas instruccionales se ajustan a las evidencias científicas que existen en nuestra lengua en torno a la instrucción en conciencia fonológica. 
En base a las evidencias científicas que existen en este ámbito en nuestra lengua, se establece como hipótesis que la práctica instruccional del profesorado para el desarrollo de la conciencia fonológica se ajusta a las mismas. Así, se espera una mayor instrucción en los niveles de rima y sílaba al inicio del $2^{\circ}$ ciclo de la Educación Infantil (3 y 4 años) para introducir la instrucción en fonemas cuando el alumnado tiene 5 años. A su vez, se espera que la instrucción en el nivel de rima y sílaba se trabaje mediante tareas de identificación y recuento y sin necesidad de apoyos visuales o escritos. Asimismo, se espera que la instrucción en conciencia fonémica se combine con la enseñanza de las reglas de conversión mediante el uso de material manipulable y que se trabaje mediante tareas de segmentación, identificación, adición y omisión de fonemas.

\section{MÉTODO}

\section{PARTICIPANTES}

Participaron 113 maestras del $2^{\circ}$ ciclo de Educación Infantil pertenecientes a 40 centros educativos de la Provincia de León (España). Del total de las maestras participantes, 28 ejercían su docencia con alumnado de 3 años (24.8\%), 42 lo hacían con alumnado de 4 años (37.2\%), 41 con alumnado de 5 años $(36.3 \%)$ y en dos casos se desconoce esta información (1.8\%). Por lo tanto, en los análisis en función del curso se tomó como muestra total 111 maestras. Respecto al tipo de centro educativo, 67 ejercían su docencia en colegios de titularidad pública (59.3\%), mientras que 44 lo hacían en colegios concertados (38.9\%), y al igual que sucede con el curso, en dos de las maestras participantes se desconoce este dato (1.8\%). El $100 \%$ de los participantes eran mujeres.

También participaron en el estudio, como observadores, 113 estudiantes del último curso del Grado en Educación Infantil quienes, tras recibir formación previa en el ámbito de la conciencia fonológica, realizaron una observación participante de la práctica del profesorado en su grupo clase. Cada uno de estos observadores se correspondió con una de las maestras participantes en el estudio.

\section{INSTRUMENTO DE MEDIDA}

Para la recogida de datos, los observadores utilizaron, para cada nivel de la conciencia fonológica (rima, sílaba y fonema) una hoja de registro independiente. En cada hoja de registro, los observadores debían anotar los ejemplos de actividades que se trabajaron durante el periodo de observación de ese nivel concreto. Además, debían registrar de forma específica el tipo y las características de los materiales que se utilizaban para el desarrollo de cada actividad, así como de las técnicas y estrategias utilizadas. A partir de la recopilación y análisis de las diferentes hojas de registro se realizó, para cada nivel de la conciencia fonológica, un análisis de tres dimensiones.

En primer lugar, se analizaron los tipos de actividades que se realizaban en clase. Así, se diferenciaron siete tipos de actividades: actividad de identificación, que exige por parte del alumnado el reconocimiento del segmento trabajado; actividad de recuento, donde el alumnado, además de identificar los segmentos trabajados, debía contar los mismos; actividad de segmentación en la que el alumnado debía fragmentar la palabra en segmentos; actividad de combinación, que consiste en combinar diferentes segmentos para formar una palabra; y actividades de adición, omisión y sustitución, enfocadas a la creación de nuevas palabras mediante la adición, omisión o sustitución de segmentos respectivamente.

A su vez, en cada uno de los tipos de actividades se analizó el grado de dificultad en el diseño de la actividad, diferenciando entre tareas de juicio, donde el alumnado simplemente debía reconocer el segmento trabajado, o tareas de producción en las que el alumnado además de reconocer el segmento debía pronunciarlo oralmente.

El segundo nivel de análisis de la práctica instruccional correspondió a los tipos de materiales de apoyo o andamiaje utilizados por el profesorado, diferenciándose entre 
material auditivo (poemas, canciones, cuentos), material visual (dibujos, apoyo escrito del segmento trabajado), material manipulativo (letras de plástico o cartón, nubes con sílabas), y apoyo de la grafomotricidad (escritura por parte del alumno de la palabra o segmento trabajado).

El tercer nivel de análisis fue la secuencia instruccional utilizada por el profesorado, analizando cómo se organizaba el trabajo del alumno (a nivel de grupo aula o mediante actividades de carácter individualizado) y qué técnicas se utilizaban, diferenciándose: a) el palmeado, indicándole al alumnado que debe dar una palmada o un golpe en la mesa por cada segmento trabajado; b) el modelado, acompañando la explicación de la actividad con un ejemplo de cómo realizarla; y c) el feedback, corrigiendo posibles respuestas erróneas y mostrando una alternativa correcta.

En dicha hoja de registro se recogieron de forma complementaria datos sociodemográficos sobre el tipo de centro educativo (público o privado), el curso de Educación Infantil en el que se impartía la docencia, el género de los participantes, el número total de alumnos, el número de alumnado con NEAE, así como cuestiones generales sobre si existía alguna medida o programa de prevención de dificultades de aprendizaje de la lectura o de detección e intervención en alumnado de riesgo en el centro escolar.

\section{PROCEDIMIENTO}

La primera fase del estudio correspondió a la formación de los observadores del estudio. Si bien, como se ha descrito anteriormente, todos ellos eran estudiantes de último curso del Grado en Educación Infantil, contando por lo tanto con una formación previa en este ámbito de estudio, con el fin de garantizar la fiabilidad de la observación de los participantes, todos ellos recibieron un entrenamiento previo implementado por una de las investigadoras en dos sesiones formativas, con cinco horas de duración. En estas sesiones se formaron en los diferentes niveles de la conciencia fonológica, así como, en las tres dimensiones de análisis de la práctica que se debían observar, diferenciando y ejemplificando cada una de ellas y realizando diferentes análisis de ejemplos específicos de niveles de la conciencia fonológica para su categorización, así como de los de diferentes tipos de actividades, y de clasificación de materiales de apoyo y estrategias.

Posteriormente tuvo lugar la fase observacional del estudio. Esta se llevó a cabo durante un periodo de tres meses coincidiendo con el periodo de prácticas del alumnado en los centros educativos y correspondió a una observación participante de la práctica del profesorado en su grupo clase en relación a la instrucción en conciencia fonológica.

Una vez realizada la observación y previo a la recopilación de las hojas de registro, las dudas surgidas fueron puestas en común y resueltas por una de las investigadoras en una sesión final con los observadores.

El presente estudio fue desarrollado dentro de la asignatura obligatoria de Prácticum II del Grado en Educación Infantil de la Universidad de León. Entre las finalidades del mismo está la observación y análisis de la práctica docente por parte del alumnado, siendo una de las dimensiones a estudiar en este caso concreto el desarrollo de la conciencia fonológica por el profesorado. Los tutores del Prácticum en los correspondientes centros educativos son informados de forma extensa de las diferentes finalidades del Prácticum, ofreciendo en todo caso, su consentimiento y ayuda desinteresada para el desarrollo óptimo del mismo. No obstante, a los efectos de esta investigación, se garantizó la total confidencialidad y anonimato de todas las personas participantes en el estudio, así como el tratamiento a nivel estadístico de todos los datos recogidos en el estudio, utilizados con una finalidad meramente científica.

\section{ANÁLISIS DE DATOS}

Una vez recogidas todas las hojas de registro se procedió a la categorización de la información por parte de las investigadoras. Para ello, se identificó en primer lugar la dimensión de conciencia fonológica que se indicaba trabajar. Seguidamente, para cada 
dimensión de conciencia fonológica se analizó de forma minuciosa el tipo de actividad que se planteaba y si esta requería dar una respuesta de juicio o de producción. Finalmente, a partir de las diferentes actividades descritas se identificó el tipo de materiales y de estrategias que se empleaban. Para cada categoría de análisis, las respuestas se codificaron en Excel según la presencia o no de los aspectos mencionados.

Para el análisis de los datos se utilizó el software estadístico SPSS versión 24. Los datos recogidos fueron analizados de forma independiente para cada nivel de la conciencia fonológica que se instruía. En cada nivel se calcularon estadísticos descriptivos de porcentajes y tablas de contingencia considerando el tipo de tareas, materiales y estrategias empleadas. A su vez, se analizó la existencia de relación entre el tipo de instrucción implementada para el desarrollo de la conciencia fonológica y el curso de Educación Infantil mediante la prueba Chicuadrado, y adicionalmente, en aquellos casos en los que se obtuvo relación entre variables, se halló la magnitud de tal relación a través de la prueba $V$ de Cramer; resultados todos ellos que se presentan en el siguiente apartado.

\section{RESULTADOS}

En este apartado, se muestran en primer lugar, los resultados a nivel de rima, a continuación, los resultados a nivel de sílaba y finalmente, los resultados a nivel de fonema. En cada uno de estos niveles se muestran los resultados relativos al tipo de tarea, a los materiales y a las estrategias utilizadas, así como, los resultados del análisis en función del curso. Los porcentajes relativos al tipo de tarea, a los materiales y a las estrategias utilizadas que se muestran en las tablas para cada uno de los niveles de análisis de la conciencia fonológica (rima, sílaba y fonema), se corresponden con el total de maestras que indicaron trabajar ese nivel, y no con el total de los participantes del estudio. Asimismo, las categorías de respuesta incluidas en cada dimensión de análisis no eran excluyentes entre sí, por lo quela información procedente de un mismo docente podía categorizarse en más de una categoría de respuesta. Por ello, en las diferentes dimensiones de análisis incluidas, el sumatorio de sus respectivas categorías puede superar el 100\%.

\section{INSTRUCCIÓN EN CONCIENCIA FONOLÓGICA A NIVEL DE RIMA}

Del total de docentes que participaron en este estudio sólo el 13,3\% (n=15) trabajaron el nivel de rima de la conciencia fonológica de forma explícita. En la Tabla 1 se presenta el porcentaje de docentes para cada tipo de tareas, materiales y estrategias que utilizaron para ello.

Tal y como se observa en la Tabla 1, todas las maestras que indicaron trabajar el nivel de rima, utilizaron tareas de identificación, en las que el alumnado tenía que reconocer qué palabras compartían sonidos o rimaban con un sonido o palabra dado. Además, este tipo de actividades se trabajaron en mayor medida a partir de tareas de juicio, que requieren menor esfuerzo cognitivo por parte del alumnado, que a partir de tareas de producción donde el alumnado debía dar o producir un sonido o una palabra correcta.

En relación al tipo de material o andamiaje, el más empleado por las maestras que trabajaron el nivel de rima fue el material auditivo, siendo los poemas o trabalenguas los recursos más utilizados, seguido de las canciones. El segundo material más empleado, aunque con un porcentaje menor, fue el material visual, utilizándose en la mayoría de los casos tarjetas con dibujos o flashcards, y en muy pocos casos se hizo uso del apoyo de la palabra escrita. En ningún caso se empleó material manipulativo ni se trabajó la grafomotricidad mediante la escritura de la palabra por parte del alumnado.

Respecto a las estrategias instruccionales más empleadas (ver Tabla 1) destaca el trabajo en grupo o en asamblea, seguido del modelado y de planteamientos individuales, estos últimos referidos a situaciones en las que el alumnado trabajaba cada uno en su ficha, libro, etc. Por su parte, la estrategia de feedback fue la menos empleada por las maestras y la estrategia del palmeado no se empleó en ningún caso. 
Tabla 1

Síntesis del análisis de la instrucción en el nivel de rima

\begin{tabular}{|c|c|c|}
\hline Dimensiones & Tipo de respuesta & $\%$ \\
\hline \multicolumn{3}{|l|}{ TAREAS } \\
\hline \multirow{3}{*}{$\begin{array}{l}\text { Identificación } \\
\quad(100 \%)\end{array}$} & Juicio & $66,7 \%$ \\
\hline & Producción & $33,3 \%$ \\
\hline & Juicio-Producción & - \\
\hline \multirow{3}{*}{$\begin{array}{c}\text { Recuento } \\
(0,0 \%)\end{array}$} & Juicio & - \\
\hline & Producción & - \\
\hline & Juicio-Producción & - \\
\hline \multirow{3}{*}{$\begin{array}{c}\text { Segmentación } \\
(0,0 \%)\end{array}$} & Juicio & - \\
\hline & Producción & - \\
\hline & Juicio-Producción & - \\
\hline \multirow{3}{*}{$\begin{array}{c}\text { Combinación } \\
(0,0 \%)\end{array}$} & Juicio & - \\
\hline & Producción & - \\
\hline & Juicio-Producción & - \\
\hline \multirow{3}{*}{$\begin{array}{l}\text { Adición } \\
(0,0 \%)\end{array}$} & Juicio & - \\
\hline & Producción & - \\
\hline & Juicio-Producción & - \\
\hline \multirow{3}{*}{$\begin{array}{l}\text { Omisión } \\
(0,0 \%)\end{array}$} & Juicio & - \\
\hline & Producción & - \\
\hline & Juicio-Producción & - \\
\hline \multirow{3}{*}{$\begin{array}{c}\text { Sustitución } \\
(0,0 \%)\end{array}$} & Juicio & - \\
\hline & Producción & - \\
\hline & Juicio-Producción & - \\
\hline \multicolumn{3}{|l|}{ MATERIALES } \\
\hline \multirow{2}{*}{$\begin{array}{l}\text { Manipulativo } \\
(0,0 \%)\end{array}$} & Letras de plástico/cartón & - \\
\hline & Tarjetas/nubes de sílabas & - \\
\hline \multirow{2}{*}{$\begin{array}{l}\text { Visual } \\
(26,7 \%)\end{array}$} & Apoyo escrito de la palabra & $6,7 \%$ \\
\hline & Dibujos/flashcards & $20 \%$ \\
\hline \multirow{3}{*}{$\begin{array}{l}\text { Auditivo } \\
(60 \%)\end{array}$} & Canciones & $20 \%$ \\
\hline & Poemas & $40 \%$ \\
\hline & Cuento & - \\
\hline $\begin{array}{c}\text { Grafomotricidad } \\
(0,0 \%)\end{array}$ & Escritura de palabra por el alumno & - \\
\hline \multirow{5}{*}{ ESTRATEGIAS } & Palmeado & - \\
\hline & Modelado & $46,7 \%$ \\
\hline & Grupal/Asamblea & $80 \%$ \\
\hline & Individual & $20 \%$ \\
\hline & Feedback & $13,3 \%$ \\
\hline
\end{tabular}


En cuanto a la relación entre la práctica en el nivel de rima de la conciencia fonológica y el curso, no se obtuvo relación significativa entre variables $(p=, 696)$, indicando que el hecho de que instruyan o no en el nivel de rima no depende del curso. Respecto al tipo de actividades empleadas, no ha sido posible calcular ningún estadístico dado que ninguna maestra indicó utilizarlas en ninguno de los cursos, a excepción de la tarea de identificación que es empleada por todas las maestras que indicaron trabajar el nivel de rima. Lo mismo ocurre con el material manipulativo y con la grafo-motricidad, así como con las estrategias del palmeado. Respecto al resto de materiales y estrategias, tampoco se obtuvo relación con el curso (material auditivo, $p=, 961$; material visual, $p=585$; estrategia de modelado, $p=$ ,330; estrategia grupal, $p=, 788$; estrategia individual, $p=$,788; y estrategia de feedback, $p=$,719), por lo que la utilización de este tipo de materiales y estrategias no depende del curso de Educación Infantil en el que se enseña.

\section{INSTRUCCIÓN EN CONCIENCIA FONOLÓGICA A NIVEL DE SÍLABA}

Del total de docentes participantes en el estudio un 70,8\% ( $n=80)$ trabajaron la conciencia silábica. En la Tabla 2 se presenta el porcentaje de docentes para cada tipo de tareas, materiales y estrategias que utilizaron para ello.

Como se puede observar en la Tabla 2, el tipo de tareas utilizadas en mayor porcentaje por los docentes que trabajaron la conciencia silábica fueron, en primer lugar, las tareas de identificación seguidas de las tareas de recuento. En ambos casos predominaron las actividades de juicio, donde el alumnado tenía que indicar si las sílabas sonaban iguales o distintas, o el número de sílabas que contenía una palabra dada. A este tipo de tareas le siguen las tareas de segmentación, las tareas de combinación y las tareas de adición, predominando en todos los casos las actividades de producción. Así, en el caso de la segmentación silábica, los docentes

Tabla 2

Síntesis del análisis de la instrucción en el nivel de sílaba

\begin{tabular}{|c|c|c|}
\hline Dimensiones & Tipo de respuesta & $\%$ \\
\hline \multicolumn{3}{|l|}{ TAREAS } \\
\hline \multirow{3}{*}{$\begin{array}{l}\text { Identificación } \\
(67,6 \%)\end{array}$} & Juicio & $45 \%$ \\
\hline & Producción & $11,3 \%$ \\
\hline & Juicio-Producción & $11,3 \%$ \\
\hline \multirow{3}{*}{$\begin{array}{l}\text { Recuento } \\
(61,3 \%)\end{array}$} & Juicio & $37,5 \%$ \\
\hline & Producción & $5 \%$ \\
\hline & Juicio-Producción & $18,8 \%$ \\
\hline \multirow{3}{*}{$\begin{array}{c}\text { Segmentación } \\
(33,8 \%)\end{array}$} & Juicio & $8,8 \%$ \\
\hline & Producción & $22,5 \%$ \\
\hline & Juicio-Producción & $2,5 \%$ \\
\hline \multirow{3}{*}{$\begin{array}{c}\text { Combinación } \\
(23,8 \%)\end{array}$} & Juicio & $7,5 \%$ \\
\hline & Producción & $16,3 \%$ \\
\hline & Juicio-Producción & - \\
\hline \multirow{3}{*}{$\begin{array}{l}\text { Adición } \\
(22,6 \%)\end{array}$} & Juicio & $6,3 \%$ \\
\hline & Producción & $15 \%$ \\
\hline & Juicio-Producción & $1,3 \%$ \\
\hline
\end{tabular}


Tabla 2 (Continuación)

Síntesis del análisis de la instrucción en el nivel de sílaba

\begin{tabular}{|c|c|c|}
\hline Dimensiones & Tipo de respuesta & $\%$ \\
\hline \multicolumn{3}{|l|}{ TAREAS } \\
\hline \multirow{3}{*}{$\begin{array}{l}\text { Omisión } \\
(5 \%)\end{array}$} & Juicio & $1,2 \%$ \\
\hline & Producción & $3,8 \%$ \\
\hline & Juicio-Producción & - \\
\hline \multirow{3}{*}{$\begin{array}{c}\text { Sustitución } \\
(1,2 \%)\end{array}$} & Juicio & $1,2 \%$ \\
\hline & Producción & - \\
\hline & Juicio-Producción & - \\
\hline \multicolumn{3}{|l|}{ MATERIALES } \\
\hline \multirow{2}{*}{$\begin{array}{l}\text { Manipulativo } \\
(10 \%)\end{array}$} & Letras de plástico/cartón & - \\
\hline & Tarjetas/nubes de sílabas & $10 \%$ \\
\hline \multirow{2}{*}{$\begin{array}{l}\text { Visual } \\
(57,5 \%)\end{array}$} & Apoyo escrito de la palabra & $42,5 \%$ \\
\hline & Dibujos/flashcards & $15 \%$ \\
\hline \multirow{3}{*}{$\begin{array}{l}\text { Auditivo } \\
(7,5 \%)\end{array}$} & Canciones & $7,5 \%$ \\
\hline & Poemas & - \\
\hline & Cuento & - \\
\hline $\begin{array}{l}\text { Grafomotricidad } \\
(10 \%)\end{array}$ & Escritura de palabra por el alumno & $10 \%$ \\
\hline \multirow{5}{*}{ ESTRATEGIAS } & Palmeado & $60 \%$ \\
\hline & Modelado & $35 \%$ \\
\hline & Grupal/Asamblea & $86,3 \%$ \\
\hline & Individual & $35 \%$ \\
\hline & Feedback & $16,3 \%$ \\
\hline
\end{tabular}

que trabajaron esta dimensión emplearon actividades de producción donde el alumnado debía dividir fonológicamente la palabra dada en las sílabas correspondientes. Respecto a la combinación silábica, las actividades de producción consistían en actividades donde el alumnado debía reproducir una palabra mediante la unión de sus sílabas. Finalmente, respecto a la adición de sílabas los docentes utilizaron actividades de producción en las que el alumnado debía reproducir las palabras resultantes de añadir determinadas sílabas a la misma. Por último, las tareas menos utilizadas por los docentes para la instrucción sobre el nivel de sílaba fueron las tareas de sustitución y las tareas de omisión.
Respecto al tipo de material (ver Tabla 2), el más utilizado por las maestras que trabajaron el nivel de sílaba fue el material visual, en mayor medida mediante apoyo de la palabra escrita y, en menor medida a través de dibujos o flashcards. Respecto al resto de materiales, muy pocos docentes emplearon material manipulativo, material auditivo y la escritura de la palabra por parte del alumnado (grafomotricidad).

Finalmente, para trabajar la conciencia silábica, el tipo de estrategias instruccionales más empleadas por el profesorado fueron el trabajo en grupo o en asamblea, donde se trabajaba a nivel de grupo clase y todos los niños participaban de un modo $u$ otro, y el palmeado, donde el alumnado debía dar 
golpes sobre la mesa o palmas mientras segmentaban o contaban el número de sílabas de una palabra. Por su parte, las estrategias instruccionales menos empleadas para trabajar la sílaba fueron el modelado y el trabajo individual a través de fichas, y muy pocos docentes utilizaron la técnica del feedback.

En cuanto a la relación entre el curso y el hecho de que instruyan o no en el nivel de sílaba se obtuvo una baja relación entre ambas variables $(p=, 015 ; \mathrm{V}$ de Cramer $=$ ,28). Concretamente, en Educación Infantil de 3 años los docentes instruyen menos en la dimensión de sílaba $(17,7 \%)$ frente a los docentes de Educación Infantil de 4 y 5 años (40,5\% y $41,8 \%$ respectivamente).

Sin embargo, en cuanto al tipo de tarea, material y estrategias empleadas para instruir en la dimensión de sílaba, no se obtuvo relación con el curso de Educación Infantil, a excepción de la estrategia de planteamientos individuales donde se obtuvo una baja relación ( $p=$,048; $V$ de Cramer $=, 28)$, indicando que la sílaba se trabaja en menor medida mediante actividades y planteamientos individuales en Educación Infantil de 3 años $(3,5 \%)$ que en Educación Infantil de 4 y 5 años ( $50 \%$ y $46,5 \%$ respectivamente).

\section{INSTRUCCIÓN EN CONCIENCIA FONOLÓGICA A NIVEL DE FONEMA}

Del total de profesorado participante en el estudio un $87,6 \%(n=99)$ de los casos trabajaron el nivel de conciencia fonémica con su alumnado. En la Tabla 3 se presenta el porcentaje de docentes para cada tipo de tareas, materiales y estrategias utilizadas.

Como se puede observar en la Tabla 3, la tarea más empleada en la instrucción en conciencia fonémica fue la tarea de identificación, con un uso similar entre las actividades de juicio y de producción. Seguidamente, se observan las tareas de combinación, de recuento y de segmentación. Respecto a las tareas de recuento predominó el uso de actividades de juicio, mientras que respecto a las tareas de combinación y segmentación predominaron las actividades

Tabla 3

Síntesis del análisis de la instrucción en el nivel de fonema

\begin{tabular}{|c|c|c|}
\hline Dimensiones & Tipo de respuesta & $\%$ \\
\hline \multicolumn{3}{|l|}{ TAREAS } \\
\hline \multirow{3}{*}{$\begin{array}{c}\text { Identificación } \\
(96 \%)\end{array}$} & Juicio & $30,3 \%$ \\
\hline & Producción & $24,2 \%$ \\
\hline & Juicio-Producción & $41,4 \%$ \\
\hline \multirow{3}{*}{$\begin{array}{l}\text { Recuento } \\
(16,2 \%)\end{array}$} & Juicio & $12,2 \%$ \\
\hline & Producción & - \\
\hline & Juicio-Producción & $4 \%$ \\
\hline \multirow{3}{*}{$\begin{array}{c}\text { Segmentación } \\
(11,1 \%)\end{array}$} & Juicio & $1 \%$ \\
\hline & Producción & $10,1 \%$ \\
\hline & Juicio-Producción & - \\
\hline \multirow{3}{*}{$\begin{array}{c}\text { Combinación } \\
(18,2 \%)\end{array}$} & Juicio & $2 \%$ \\
\hline & Producción & $15,2 \%$ \\
\hline & Juicio-Producción & $1 \%$ \\
\hline \multirow{3}{*}{$\begin{array}{l}\text { Adición } \\
\text { (7\%) }\end{array}$} & Juicio & $3 \%$ \\
\hline & Producción & $3 \%$ \\
\hline & Juicio-Producción & $1 \%$ \\
\hline
\end{tabular}


Tabla 3 (Continuación)

Síntesis del análisis de la instrucción en el nivel de fonema

\begin{tabular}{|c|c|c|}
\hline Dimensiones & Tipo de respuesta & $\%$ \\
\hline \multicolumn{3}{|l|}{ TAREAS } \\
\hline \multirow{3}{*}{$\begin{array}{l}\text { Omisión } \\
\text { (1\%) }\end{array}$} & Juicio & - \\
\hline & Producción & $1 \%$ \\
\hline & Juicio-Producción & - \\
\hline \multirow{3}{*}{$\begin{array}{l}\text { Sustitución } \\
\text { (4\%) }\end{array}$} & Juicio & - \\
\hline & Producción & $4 \%$ \\
\hline & Juicio-Producción & - \\
\hline \multicolumn{3}{|l|}{ MATERIALES } \\
\hline \multirow{2}{*}{$\begin{array}{l}\text { Manipulativo } \\
(14,1 \%)\end{array}$} & Letras de plástico/cartón & $12,1 \%$ \\
\hline & Tarjetas/nubes de sílabas & $2 \%$ \\
\hline \multirow{2}{*}{$\begin{array}{l}\text { Visual } \\
(75,8 \%)\end{array}$} & Apoyo escrito de la palabra & $59,6 \%$ \\
\hline & Dibujos/flashcards & $16,2 \%$ \\
\hline \multirow{3}{*}{$\begin{array}{l}\text { Auditivo } \\
(16,2 \%)\end{array}$} & Canciones & $5,1 \%$ \\
\hline & Poemas & - \\
\hline & Cuento & $11,1 \%$ \\
\hline $\begin{array}{c}\text { Grafomotricidad } \\
(12,1 \%)\end{array}$ & Escritura de palabra por el alumno & $12,1 \%$ \\
\hline \multirow{5}{*}{ ESTRATEGIAS } & Palmeado & $1 \%$ \\
\hline & Modelado & $45,5 \%$ \\
\hline & Grupal/Asamblea & $88,9 \%$ \\
\hline & Individual & $37,4 \%$ \\
\hline & Feedback & $23,2 \%$ \\
\hline
\end{tabular}

de producción. Las tareas menos utilizadas por los docentes para instruir sobre el nivel fonémico fueron las tareas de adición, sustitución y omisión.

Atendiendo al tipo de material (ver Tabla 3), el más utilizado por los docentes que indicaron trabajar el nivel fonémico fue el material visual, fundamentalmente mediante el apoyo visual de la palabra escrita, aunque también mediante dibujos o flashcards. El siguiente material más empleado, aunque con un porcentaje menor, fue el material auditivo, fundamentalmente a partir de la narración de un cuento. A este le siguen el material manipulativo, predominando en este caso el uso de letras de plástico o cartón, y la escritura de palabras o fragmentos silábicos por parte del alumnado.

Respecto a las estrategias instruccionales, la más empleada por los docentes que instruían en el nivel fonémico, fue el trabajo grupal, donde el alumnado trabajaba de forma conjunta o en asambleas. A esta le siguen, aunque con menor porcentaje, la técnica del modelado, el trabajo individual donde cada alumno trabajaba en su ficha o libro, y la técnica del feedback. Respecto al palmeado, casi ningún docente indicó emplear esta técnica instruccional para trabajar el nivel fonémico.

En cuanto a la relación entre la práctica desarrollada a nivel de conciencia fonémica y 
el curso sólo se obtuvo una relación baja entre el curso y las estrategias del trabajo grupal ( $p$ $=, 036 ; V$ de Cramer $=, 26)$ e individual ( $p$ $=, 015 ; \mathrm{V}$ de Cramer $=, 29)$. Por tanto, se puede considerar que en Educación Infantil de 3 años se trabajan los fonemas en mayor medida de forma grupal $(31,4 \%)$ y en menor medida de forma individual (13,8\%), mientras que en Educación Infantil de 5 años ocurre el caso contrario, trabajando los fonemas en mayor medida de forma individual (50\%) y en menor medida de forma grupal $(30,2 \%)$.

\section{DISCUSIÓN}

El objetivo del presente estudio fue analizar la práctica instruccional en una muestra de maestras del $2^{\circ}$ ciclo de Educación Infantil de la Provincia de León (España) en torno a la instrucción en conciencia fonológica, con el fin de valorar su ajuste a los conocimientos derivados de la investigación científica sobre qué prácticas instruccionales resultan efectivas para la mejora de la conciencia fonológica. En base a los resultados obtenidos es posible extraer las siguientes conclusiones.

Una primera conclusión del estudio es que no existe una relación significativa entre la práctica instruccional para desarrollar la conciencia fonológica del alumnado y el curso específico de Educación Infantil, 3, 4 o 5 años. En concreto, la única relación obtenida fue que los docentes de Educación Infantil de 4 y 5 años trabajan en mayor medida la dimensión silábica de la conciencia fonológica frente a los de Educación Infantil de 3 años. En este sentido, las evidencias científicas a nivel empírico han mostrado que la instrucción en el nivel de rima y en el nivel de sílaba debe comenzar antes de que el alumnado tenga 5 años (Domínguez, 1994, 1996a; Gimeno et al., 1994), introduciendo la instrucción a nivel de fonemas con alumnado de 5 años (Cuadro y Trías, 2008; Ramos y Cuadrado, 2003). Por tanto, parece necesario que la conciencia silábica se trabaje en mayor medida en Educación Infantil de 3 años introduciendo un mayor grado de andamiaje y mediante actividades de menor dificultad como las de juicio, y que en Educación Infantil de 5 años se trabaje principalmente la conciencia fonémica en combinación con la enseñanza de las reglas de conversión mediante el uso de material manipulable.

En segundo lugar, respecto a la instrucción centrada en el desarrollo de la conciencia fonológica a nivel de rima, todos los participantes indicaron instruir en esta dimensión mediante la tarea de identificación, fundamentalmente con actividades de juicio; lo cual resulta efectivo para favorecer las habilidades metafonológicas previas a la lectura (Domínguez, 1994). Asímismo, el material más empleado fue de tipo auditivo (poemas, canciones, etc.), aspecto que favorece el desarrollo de la conciencia fonológica a nivel de rima gracias a las semejanzas que se dan entre palabras (Herrera, Defior y Lorenzo, 2007). Respecto a las estrategias instruccionales empleadas en esta dimensión, predominan los planteamientos grupales y la técnica del modelado; estrategias que resultan efectivas para favorecer la eficacia de la instrucción en conciencia fonológica (Domínguez, 1994). Por tanto, a pesar de ser la dimensión menos trabajada por el profesorado, se podría considerar que el tipo de actividades, los materiales y las estrategias que emplean para su instrucción se ajustan a los resultados derivados de la investigación científica.

Respecto a la instrucción implementada para trabajar la conciencia fonológica a nivel de sílaba, las tareas que predominan son de recuento e identificación, fundamentalmente a partir de tareas de juicio, tareas que son adecuadas para trabajar este nivel ya que exigen menos recursos cognitivos por parte del alumnado (Gimeno et al., 1994). También se observa la presencia de tareas de segmentación, tarea que resulta efectiva cuando el alumnado presenta dificultades de aprendizaje, al menos para mejorar las habilidades fonológicas, ya que no se consiguen efectos sobre la lectura (Rueda y Sánchez, 1996). Por otro lado, el tipo de material más empleado fue de tipo visual, fundamentalmente mediante el apoyo escrito de la palabra o el segmento trabajado. En este sentido, a pesar de que los resultados de la investigación muestran que el apoyo de 
la palabra escrita no resulta necesario para mejorar las habilidades fonológicas (Gimeno et al., 1994), se desconocen los efectos sobre la lectura. Finalmente, respecto a las estrategias instruccionales, las más empleadas son el trabajo de forma grupal y la técnica del palmeado; favoreciendo esta última el análisis fonológico (Olson, 1990). Cabe destacar el trabajo de forma individualizada, ya que fue la única variable que presentó relación con el curso, indicando que esta forma de trabajo se empleó en menor medida por docentes de Educación Infantil de 3 años frente a los docentes de 4 y 5 años. Por tanto, se podría considerar que el tipo de actividades, los materiales y las estrategias que el profesorado emplea para trabajar esta dimensión, se ajustan a las evidencias derivadas de la investigación científica.

Finalmente, en relación a la instrucción centrada en el nivel fonémico, el tipo de tarea que predomina es la de identificación, observándose una baja frecuencia de uso en las tareas de segmentación, combinación, adición, sustitución y omisión, siendo precisamente estas últimas las tareas que resultan más efectivas para la mejora de la lectura (Cuadro y Trías, 2008; Domínguez, 1994, 1996a; González et al., 2013; Ramos y Cuadrado, 2003). Por tanto, parece necesario que las tareas de segmentación, combinación, adición, sustitución y omisión de fonemas se utilicen en mayor medida cuando se trabaje la conciencia fonémica con alumnado de Educación Infantil de 5 años. Respecto al tipo de material empleado, se destaca el de tipo visual, fundamentalmente mediante el apoyo escrito de la palabra, que favorece el aprendizaje de las reglas de conversión; aspecto que resulta esperanzador por ser ambas dimensiones, conciencia fonológica y reglas de conversión, fundamentales para el acceso al código escrito (Jiménez y O Shanahan, 2008). Sin embargo, sólo un escaso número de docentes empleó material manipulativo para trabajar los fonemas, siendo este efectivo para favorecer la relación entre fonemas y grafemas, mejorando a su vez la lectura (Defior, 2008; Domínguez, 1994; González et al., 2013; Ramos y Cuadrado,
2003); por lo que sería necesario utilizar con mayor frecuencia material manipulativo cuando se trabaja la dimensión fonémica de la conciencia fonológica. Finalmente, respecto a las estrategias instruccionales, la técnica más empleada para trabajar la conciencia fonémica es el modelado, que resulta efectiva para favorecer la eficacia de la instrucción en conciencia fonológica (Domínguez, 1994). También se destacan los planteamientos tanto individuales como de trabajo en grupo. Sin embargo, en ambos planteamientos se obtuvo relación con el curso, indicando que los docentes de 3 años tienden a trabajar la conciencia fonémica en mayor medida mediante actividades grupales, mientras que los docentes de 4 y 5 años plantean en mayor medida el trabajo mediante fichas o actividades de carácter individualizado; siendo necesario plantear en mayor medida tareas colaborativas cuando se trabajan los fonemas con alumnado de Educación Infantil de 5 años. Por tanto, se podría considerar que el planteamiento instruccional del profesorado de Educación Infantil, para trabajar la dimensión fonémica presenta desajustes con la investigación científica, derivados principalmente del tipo de tarea que se emplea, pero también de los materiales y las técnicas que se utilizan. Estos resultados son alarmantes dado que es el nivel fonémico el que mayor efecto ejerce sobre el aprendizaje lector inicial (Defior y Serrano, 2011 ; Rabazo y Moreno, 2004).

Las conclusiones ofrecidas en este estudio, deben interpretarse con cautela, dentro de los límites impuestos por el mismo. Uno de ellos está ligado a la representatividad de la muestra seleccionada en este estudio, por lo que resultaría interesante desarrollar estudios futuros en esta línea con mayores tamaños muestrales y abarcando otras provincias españolas. Otra limitación deriva del instrumento de recogida de datos utilizado, que se limitó al registro de los aspectos trabajados por el profesorado en torno a la conciencia fonológica, pero no contempló la frecuencia con la que los profesores utilizaban dichas prácticas. Así, se aconsejaría utilizar en futuros estudios protocolos de 
observación diseñados previamente en base a cada una de las dimensiones a observar que permitan registrar la frecuencia de las respuestas observadas. También, se podría complementar esta observación con el análisis de las programaciones didácticas o los planes de trabajos diseñados. Otra posible limitación del estudio estuvo ligada al hecho de optar por una observación directa y participante, lo que hizo imposible el realizar un doble análisis de la práctica del profesorado que posibilitara el cálculo del acuerdo interjueces como dato de fiabilidad de la medición. No obstante, el hecho de que los observadores contaran con amplia formación previa en este ámbito, unido a las sesiones formativas previas en las que se discutieron, analizaron, codificaron ejemplos concretos en cada dimensión de análisis a observar, así como las sesiones de discusión de resultados y puestas en común con una de las investigadoras, parece suficiente para poder garantizar la fiabilidad de la medida. Otro aspecto interesante a abordar en futuros estudios consistiría en analizar si la práctica implementada por el profesorado en torno al desarrollo de la conciencia fonológica se relaciona con el rendimiento lector del alumnado.

A pesar de las limitaciones descritas, los resultados del presente estudio apoyan lo establecido por estudios previos que han mostrado que los docentes de Educación Infantil y Educación Primaria no poseen un profundo conocimiento científico necesario para implementar en el aula una práctica instruccional basada en la evidencia empírica (Guzmán, Correa, Arvelo y Abreu, 2015); con las consecuencias negativas que ello puede suponer en el rendimiento del alumnado dado que, como han señalado estudios previos, el enfoque de enseñanza del docente está estrechamente relacionado con el rendimiento del alumnado (Robledo, Fidalgo y Méndez, 2019). Asimismo, se ha señalado que los docentes de Educación Infantil y Educación Primaria manifiestan la necesidad de recibir asesoramiento pedagógico, fundamentalmente en cuestiones relacionadas con metodologías didácticas y atención a la diversidad, concretamente en el ámbito de las dificultades de aprendizaje (Anaya, Suárez y Pérez, 2009). Por tanto, desde el ámbito educativo parece necesario el desarrollar programas de desarrollo profesional del profesorado en torno al dominio de prácticas instruccionales empíricamente validadas para la enseñanza de la lectura acordes a las evidencias científicas derivadas de los estudios de meta-análisis (Ripoll y Aguado, 2014). En este sentido y considerando la evidencia empírica existente, la práctica docente para la enseñanza no sólo de la lectura, sino también de la escritura, debe comenzar con una enseñanza explícita y sistemática de los procesos de bajo nivel cognitivo en los primeros años de escolarización (Alves et al., 2016; González et al., 2013), y, una vez automatizados los mismos, se debe promover el desarrollo de los procesos de alto nivel cognitivo mediante enfoques estratégicos y autorregulados (Arrimada, Torrance y Fidalgo, 2019; Fidalgo, Torrance, Arias y Martínez, 2014; Fidalgo, Torrance, y Robledo, 2011 ; Gottheil et al., 2019); enfoque instruccional que se ha mostrado efectivo para la mejora del rendimiento del alumnado (Rodríguez, Piñeiro, Regueiro, Estévez y Val, 2017; SáizManzanares y Queiruga-Dios, 2018).

De esta forma, si se le proporciona al profesorado formación adicional para la implementación de una práctica instruccional basada en la evidencia empírica, se contribuiría a descartar como causa de las dificultades de aprendizaje una instrucción de baja calidad; en línea con el enfoque preventivo propuesto por el modelo de respuesta a la intervención (Arrimada et al., 2018)

\section{- Conflicto de intereses.}

Los autores declaran no tener ningún conflicto de intereses.

\section{- Agradecimientos}

Esta investigación no habría sido posible sin la labor inestimable de observación participante del alumnado del Grado en Educación Infantil de la Universidad de León de la promoción participante y la implicación desinteresada y altruista del profesorado tutor del Prácticum de dicha especialidad de los centros educativos participantes.

Durante el desarrollo de esta investigación se recibieron ayudas del Ministerio de Economía y Competitividad, EDU201567484-P (MINECO/FEDER), proyecto de investigación concedido a la segunda autora. 


\section{REFERENCIAS}

Alves, R., Limpo, T., Fidalgo, R., Carvalhais, L., Alvares, L., \& Castro, S. (2016). The impact of promoting transcription on early text production: effects on bursts and pauses, levels of written language, and writing performance. Journal of Educational Psychology, 108(5), 665-679. doi:10.1037/edu0000089

Anaya, D., Suárez, J. M., \& Pérez, J. C. (2009). Necesidades de asesoramiento o ayuda de expertos demandadas por el profesorado de educación infantil, primaria y secundaria. Revista de Investigación Educativa, 27(2), 413-425.

Arnáiz, P., Castejón, J. L., Ruiz, Ma . S., \& Guirao, J. M. (2002). Desarrollo de un programa de habilidades fonológicas y su implicación en el acceso inicial a la lectoescritura en alumnos de segundo ciclo de educación infantil. Educación, Desarrollo y Diversidad, 5, 29-51.

Arrimada, M., Torrance, M., \& Fidalgo, R. (2018). Supporting first-grade writers who fail to learn: multiple single-case evaluation of a Response to Intervention approach. Reading and Writing, 37(4), 865-891. doi:10.1007/s $11145-018-9817-x$

Arrimada, M., Torrance, M., \& Fidalgo, R. (2019). Effects of teaching planning strategies to first-grades writers. British Journal of Educational Psychology, 89(4), 670-688. doi:10.1111/bjep.12251

Bravo, L., Villalón, M., \& Orellana, E. (2006). Predictibilidad del rendimiento en la lectura: una investigación de seguimiento entre primer y tercer año. Revista Latinoamericana de Psicología, 38(1), 9-20.

Castles, A., \& Coltheart, M. (2004). Is there a casual link from phonological awareness to success in learning to read? Cognition, 91, 77-111. doi:10.1016/50010. 0277(03)00164-1.

Cuadro, A., \& Trías, D. (2008). Desarrollo de la conciencia fonémica: evaluación de un programa de intervención. Revista Argentina de Neuropsicología, 11, 1-8.

Defior, S. (2008). ¿Cómo facilitar el aprendizaje inicial de la lectoescritura? Papel de las habilidades fonológicas.
Infancia y Aprendizaje, 37 (3), 333-345. doi: $10.1174 / 021037008785702983$.

Defior, S., \& Serrano, F. (201 1). La conciencia fonémica, aliada de la adquisición del lenguaje escrito. Revista de Logopedia, Foniatría y Audiología, 31(1), 2-13. doi:10.1016/S0214-4603(11)70165-6.

Defior, S., \& Tudela, P. (1994). Effect of phonological training on reading and writing acquisition. Reading and Writing: An Interdisciplinary Journal, 6, 299-320. doi: 10.1007/bf01027087

Domínguez, A. B. (1994). Cómo prevenir algunas de las dificultades del aprendizaje de la lectura y de la escritura. Enseñanza XII, 51, 167-179.

Domínguez, A. B. (1996a). El desarrollo de habilidades de análisis fonológico a través de programas de enseñanza. Infancia y aprendizaje, 76, 69-81. doi:10.1174/0210 37096762905562.

Domínguez, A. B. (1996b). Evaluación de los efectos a largo plazo de la enseñanza de habilidades de análisis fonológico en el aprendizaje de la lectura y de la escritura. Infancia y aprendizaje, 76, 83-96. doi: $10.1174 / 021037096762905571$

Fidalgo, R., \& Robledo, P. (2010). El ámbito de las dificultades específicas de aprendizaje en España a partir de la Ley Orgánica de Educación. Papeles de Psicólogo, $31(2)$, $171-182$.

Fidalgo, R., Torrance, M., Arias, O., \& Martínez, B. (2014). Comparison of reading-writing patterns and performance of students with and without reading difficulties. Psicothema, 26(4), 442-448. doi:10.7334/ psicothema2014.23

Fidalgo, R., Torrance, M., \& Robledo, P. (2011). Comparación de dos programas de instrucción estratégica y autorregulada para la mejora de la composición escrita. Psicothema, 23(4), 672-680.

Fuchs, D., Compton, D. L., Fuchs, L. S., Bryant, V. J., Hamlett, C. L., \& Lambert, W. (2012). First-grade cognitive abilities as long-term predictors of reading comprehension and disability status. Journal of Learning Disabilities, 45(3), 217-231. doi: $10.1177 / 0022219412442154$

Fuchs, D., Fuchs, L., \& Compton, D. L. (2012). Smart RTI: a next-generation 
approach to multilevel prevention. Council for Exceptional Children. 78(3), 263-279. doi:10.1177/001440291207800301

Gimeno, A., Clemente, A., López, T., \& Castro, A. (1994). ¿Cómo mejorar la conciencia fonológica en la escuela? Descripción y análisis de un programa de intervención. Comunicación, lenguaje y educación, 21, 8795. doi:10.1174/021470394321513898

González, R. M., López, S., Vilar, J., \& Rodríguez, A. (2013). Estudio de los predictores de la lectura. Revista de Investigación en Educación, 17 (2), 98-110.

Gottheil, B., Brenlla, M. E., Barreyro, J. P., Pueyrredón, D., Aldrey, A., Buonsanti, L, Freire, L., Rossi, A. I., \& Molina, S. (2019). Eficacia del programa "Lee Comprensivamente" para la enseñanza de estrategias de comprensión lectora en estudiantes de Educación Primaria. Revista de Psicología y Educación, 14(2), 99 111. doi:10.23923/rpye2019.01.175

Guzmán, R., Correa, A. D., Arvelo, C. N., \& Abreu, B. (2015). Conocimiento del profesorado sobre las dificultades específicas de aprendizaje en lectura y escritura. Revista de Investigación Educativa, 33(2), 289-302. doi:10.6018/rie.33.2.211101.

Hernández-Valle, I., \& Jiménez, J. (2001). Conciencia fonémica y retraso lector: 己̇es determinante la edad en la eficacia de la intervención? InfanciayAprendizaje, 24(3),379395. doi: $10.1174 / 021037001316949284$.

Herrera, L., Defior, S., \& Lorenzo, O. (2007). Intervención educativa en conciencia fonológica en niños prelectores de lengua materna española y tamazight. Comparación de dos programas de entrenamiento. Infancia y Aprendizaje, 30(1), 39-54. doi: $10.1174 / 021037007779849718$

Jiménez, J. (2010). Response to Intervention (Rtl) model: a promising alternative for identifying students with learning disabilities? Psicothema, 22(4), 932-934.

Jiménez, J. E., Guzmán, R., Ortiz, R., Díaz, A., Estévez, A., García, E, Hernández-Valle, I., Muñetón, M., Naranjo, F., Rodrigo, M., Rodríguez, C., \& Rojas, E. (2009). Validez discriminante de la batería multimedia SICOLE-R-Primaria para la evaluación de procesos cognitivos asociados a la dislexia. Revista de Investigación Educativa, 27(1), 4971.
Jiménez, J., \& O'Shanahan, I. (2008). Enseñanza de la lectura: de la teoría y la investigación a la práctica educativa. Revista Iberoamericana de Educación, 45(5), 1-22.

Jiménez, J., Rodríguez, C., Guzmán, R., \& García, E. (2010). Desarrollo de los procesos cognitivos de la lectura en alumnos normolectores y alumnos con dificultades de aprendizaje. Revista de Educación, 353, 361-386.

Jiménez, J., \& Rojas, E. (2008). Efectos del videojuego Tradislexia en la conciencia fonológica y reconocimiento de palabras en niños disléxicos. Psicothema, 20(3), 347 353.

National Joint Committee of Learning Disabilities. (2006). Learning disabilities and young children: identification and intervention. Learning Disability Quarterly, 30, 63-72. doi:10.1044/policy.tr200700307

Olson, M. W. (1990). Phonemic awareness and reading achievement. Research into practice. Reading Psychology, 17 (4), 347-353.

Rabazo, M. J., \& Moreno, J. M. (2004). Análisis de la investigación en conocimiento fonológico. Ciencia Psicológica, 9, 132-154.

Ramos, J. L. (2004). Conocimiento fonológico y desarrollo lectoescritor en la educación infantil. Educación XX1, 7, 169-183. doi:10.5944/educxx 1.7.1.334

Ramos, J. L., \& Cuadrado, I. (2003). Influencia causal del conocimiento fonológico en el aprendizaje inicial de la lectoescritura. Psicología Educativa, 9(2), 113-126.

Ripoll, J., \& Aguado, G. (2014). La mejora de la comprensión lectora en español: un metaanálisis. Revista de Psicodidáctica, 19(1), 27. 44. doi:10.1387/RevPsicodidact.9001

Robledo, P., Fidalgo, R., \& Méndez, M. (2019). Evaluación de la comprensión lectora a partir del análisis de la práctica del profesorado y la interacción docente-estudiante. Revista de Educación, 384, 97-120.

Rodríguez, S., Piñeiro, I., Regueiro, B., Estevez, I., \& Val, C. (2017). Estrategias cognitivas, etapa educativa y rendimiento académico. Revista de Psicología y Educación, 12(1), 19-34.

Rueda, M., \& Sánchez, E. (1996). Relación entre conocimiento fonémico y dislexia: un estudio instruccional. Cognitiva, 8(2), 215-234. doi:10.1174/021435596763003303 
Sáiz-Manzanares, M. C., \& QueirugaDios, M. A. (2018). Evaluación de estrategias metacognitivas: aplicación de métodos online. Revista de Psicología y Educación, 13(1), 33-45. doi:10.23923/ rpye2018.01.156

Simmons, D. C., Coyne, M. D., Kwok, O., McDonagh, S., Ham, B. A., \& Kame'enui, E. J. (2008). Indexing response to intervention: a longitudinal study of reading risk from kindergarten through third grade. Journal of Learning Disabilities, $41(2)$, 158-173. doi: $10.1177 / 0022219407313587$

Suárez-Coalla, P., García-de-Castro, M., \& Cuetos, F. (2013). Variables predictoras de la lectura y la escritura en castellano. Infancia y Aprendizaje, 36(1), 77-89. doi:10.1174/021037013804826537
Vanderheyden, A. M., Witt, J. C., \& Gilbertson, D. (2007). A multi-year evaluation of the effects of a Response to Intervention (RTI) model on identification of children for special education. Journal of School Psychology, 45(2), 225-256. doi:10.1016/i. isp.2006.1 1.004

Ziegler, J. C., Bertrand, D., Tóth, D., Csépe, V., Reis, A., Faísca, L, Saine, N., Lyytinen, H., Vaessen, A., \& Blomert, L. (2010). Orthographic depth and its impact on universal predictors of reading: a cross-langauge investigation. Psychological Science, 21(4), 551-559. doi: $10.1177 / 0956797610363406$ 